PAPER • OPEN ACCESS

Intrinsic broadening of the energy levels of Dirac electrons in quantum dots

To cite this article: E Ahmadi and S A Jafari 2015 J. Phys.: Conf. Ser. 603012016
You may also like

- Benefits of Live View of Bond Formation
and Alianment for Adhesive Bonding
Anaracterisation
Malik Draisey, Tony Rogers and Adnan
- Editorial
- Where were you when the sun went out?

View the article online for updates and enhancements.

The Electrochemical Society

243rd Meeting with SOFC-XVIII

Boston, MA • May 28 - June 2, 2023

Accelerate scientific discovery!
Learn More \& Register

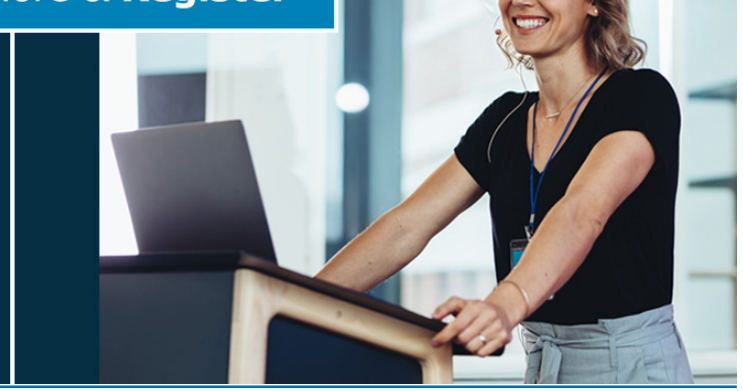

This content was downloaded from IP address 18.207 .199 .55 on $26 / 04 / 2023$ at $10: 13$ 


\title{
Intrinsic broadening of the energy levels of Dirac electrons in quantum dots
}

\author{
E Ahmadi $^{1,2}$, S A Jafari ${ }^{1,3,4}$ \\ ${ }^{1}$ Department of Physics, Sharif University of Technology, Tehran 11155-9161, Iran \\ 2 Novin Teyf Co., Tehran 14458-33834, Iran \\ ${ }^{3}$ Center of excellence for Complex Systems and Condensed Matter (CSCM), Sharif University \\ of Technology, Tehran 1458889694, Iran \\ ${ }^{4}$ School of Physics, Institute for Research in Fundamental Sciences (IPM), Tehran \\ 19395-5531, Iran \\ E-mail: jafari@physics.sharif.edu
}

\begin{abstract}
We theoretically study the quasi bound state of Dirac electrons in cylindrically symmetric quantum dots with sharp boundary. According to the existing picture, due to Klein tunneling "relativistic electrons" can not be localized by any confinement potential. We show however that despite of Klein tunneling, interference effects can cause the trapping of electron in quantum dots. Considering the quasi bound state as the state with complex energy, to find the energy of this state we solve the wave-equation with outgoing boundary condition at infinity. The imaginary part of complex energy determines the trapping time of electron within the quantum dot. We show that for any finite confining potential corresponding to any set of quantum numbers $(n, m)$ where $n$ is the principal quantum number and $m$ the magnetic quantum number, there exists a continuous band of states with finite life time. Upper and lower edges of each band corresponds to infinitely long lived states trapped inside and outside the wall of the same radius. We term this phenomenon the intrinsic broadening as it is not caused by scattering from any external potential, nor by many-body effects. This broadening appears to arise from a combination of relativistic and interference effects. The imaginary part of energy which is different for energies along the energy band is controlled by the orbital angular momentum of electron and the depth of the confining potential.
\end{abstract}

\section{Introduction}

There is a widespread picture according to which confinement of Dirac electrons in electrostatic potential, e. g. quantum dots, is impossible due to the Klein tunneling [1-2]. Therefore instead, focus has been placed on formation of confined states within quantum dots by application of perpendicular magnetic fields [3-7] or on engineering specialized devices which introduce mass terms [8-12]. This picture limits the application of quantum dots in 2D structures with Dirac energy dispersion to trapping (not localizing) Dirac electrons for long time. If the trapping time is longer than the time required for corresponding application, then one considers the electron in such confinement potential as "practically" localized electron [13-15].

In this work we find that despite the Klein tunneling, interference effects in a cylindrically symmetric quantum dot can cause infinitely long trapping time for electrons. For such long lived states, the trapping of electrons does not depend on the angular momentum and height of electrostatic potential. The quantum dot considered in this paper has cylindrically symmetric 
confinement potential for 2D Dirac electrons with sharp boundaries. We study the long lived states as quasi localized states by solving the time independent wave-equation with outgoing wave boundary condition at infinity and corresponding to complex energies. The imaginary part of the energy determines the width of broadening of energy state and trapping time of the electron within the quantum dot. If $E$ is the complex energy of localized state, then the trapping time is $\tau=\hbar / \operatorname{Im}(E)$ where $\operatorname{Im}(E)$ is imaginary part of the electron energy. For nonrelativistic quantum dots the confining potential leads to quantization of energy, that in our case is characterized by a radial principal quantum number $n$. The existing picture of the relativistic quantum dots based on graphene is that, still a discrete energy levels can be labeled by some index $n$, but it acquires an imaginary part. To find a solution whose energy has an imaginary part is to match the wave-function at the boundary and search for solutions in the complex $E$ plane. But the root-fining problem in complex plane is not as easy as the corresponding problem on the real axis. We revisit the problem of a relativistic quantum dot and search for complex energies by scanning a fine mesh of energies in the complex plane. We find that in addition to discrete energies with non-zero imaginary part, there are continuum of states centered around this solution. By moving away from this solution in both positive and negative real energy directions, we find that there are other solutions continuously connected to it whose imaginary part decreases in both directions and approach the real energy axis at two particular values that correspond to roots of Bessel functions that are regular at origin and infinity, respectively. This means that instead of a single discrete level we are dealing with a continuum of solutions that can be long lived at these two particular energies corresponding to relativistic particles confined inside and outside an infinitely deep well of the same radius.

Therefore the picture emerging from the present work is that, in the relativistic quantum dots based on graphene both the quasi bound states due to Klein tunneling and bound states in their usual quantum mechanical sense can co-exist.

\section{Theoretical calculation}

Let us first of all define what we mean by a quantum dot (QD) of Dirac electrons. The Hamiltonian of this system has a kinetic energy that is given by the Dirac Hamiltonian everywhere in the space, but the potential term is different for the interior $(\rho<R)$ and exterior $(\rho>R)$ regions of the space in a cylindrical geometry:

$$
V(\mathbf{r})=\left\{\begin{array}{lc}
0 & \rho<R \\
V_{0} & \rho>R
\end{array},\right.
$$

where potential height $V_{0}>0$, and $R$ is the radius of the quantum dot. This shape of confinement potential allows us to obtain analytical expressions for the wave-functions and facilitates the numerical calculation of the trapping time of the states of the quantum dot. The confinement potential in Eq. (1) has sharp boundaries and behavior of electron should be related to the wave function interference effects within the whole region of quantum dot. The full Hamiltonian is given by,

$$
H=v_{F}\left(\tau \sigma_{x} p_{x}+\sigma_{y} p_{y}\right)+V(\mathbf{r}),
$$

where $\tau= \pm 1$ stands for valley index, $\sigma_{j}$ denotes Pauli matrices, $p_{j}$ denotes the momentum operator that in the coordinate representation will be $-i \hbar \partial_{j} . v_{F}=3 t a / 2$ is the Fermi velocity that is given in terms of the hopping parameter $t$ and carbon-carbon bond length $a$.

The wave function $\Psi$ corresponding to Hamiltonian (2) is a two component function. Here the two components correspond to two different sub-lattices of $2 \mathrm{D}$ honeycomb lattice. For cylindrically symmetric confinement potential the two component wave function has the following form:

$$
\Psi=\left(\begin{array}{c}
\Phi_{A}(\rho) e^{i m \varphi} \\
\Phi_{B}(\rho) e^{i(m+\tau) \varphi}
\end{array}\right)
$$


where $(\rho, \varphi)$ are cylindrical coordinates and $m$ is an integer denoting the orbital angular momentum. With this form of wave function the wave-equation corresponding to the Hamiltonian in Eq. (2) is:

$$
\begin{aligned}
-i \tau \frac{d \Phi_{B}}{d \rho}-\frac{i(m+\tau)}{\rho} \Phi_{B} & =\left(\varepsilon-\nu_{0}\right) \Phi_{A}, \\
-i \tau \frac{d \Phi_{A}}{d \rho}-\frac{i m}{\rho} \Phi_{A} & =\left(\varepsilon-\nu_{0}\right) \Phi_{B} .
\end{aligned}
$$

We need to solve these equations to find energy $E$. Therefore we consider two boundary conditions: (1) Both $\phi_{A}$ and $\phi_{B}$ should be finite at $\rho=0$, and (2) we have outgoing wave form $\propto e^{i k \rho}$ at infinity. The momentum $k$ in general can be complex, and depends on the principal quantum number $n$ and angular quantum number $m$. The angular parts of the two components are given in Eq. (3). The regular solution of equations (4) and (5) inside the quantum dot $\left(V_{0}=0\right)$ is Bessel function of order $|\tau m|$ for $\phi_{A}$ and order $|\tau(m+1)|$ for $\phi_{B}$. The general solution can be written as:

$$
\left(\begin{array}{c}
\phi_{A}(\rho) \\
\phi_{B}(\rho)
\end{array}\right)=A\left(\begin{array}{c}
J_{|-\tau m|}(\varepsilon \rho / R) \\
i J_{|-\tau(m+1)|}(\varepsilon \rho / R)
\end{array}\right)
$$

where $J_{m}$ is $m^{\prime}$ th order of Bessel function and $\varepsilon=R E /$ at is dimensionless energy. Outside the quantum dot, the solutions are Hankel function of the first kind which satisfy the outgoing boundary condition at infinity. For outside of quantum dot with $\rho>R$ and $V_{0}>0$ one can write:

$$
\left(\begin{array}{c}
\phi_{A}(\rho) \\
\phi_{B}(\rho)
\end{array}\right)=C\left(\begin{array}{c}
H_{|-\tau m|}^{(1)}\left(\left(\varepsilon-\nu_{0}\right) \rho / R\right) \\
i H_{|-\tau(m+1)|}^{(1)}\left(\left(\varepsilon-\nu_{0}\right) \rho / R\right)
\end{array}\right),
$$

where $H_{n}^{(1)}$ is $n$ 'th order Hankel function of first kind and $\nu_{0}=R V_{0} /$ at is dimensionless confinement potential.

At the boundary of quantum dot the two component wave function must be continuous:

$$
\frac{H_{|-\tau m|}^{(1)}\left(\varepsilon-\nu_{0}\right)}{H_{|-\tau(m+1)|}^{(1)}\left(\varepsilon-\nu_{0}\right)}=\frac{J_{|-\tau m|}(\varepsilon)}{J_{|-\tau(m+1)|}(\varepsilon)} .
$$

From this seemingly simple equation, we can find the complex energy spectrum of graphene dot. The imaginary part of energy determines the broadening of energy state and hence the trapping of electron within quantum dot. Eq. (8) is invariant under $m \rightarrow-m$ and $\tau \rightarrow-\tau$ which means that energy spectrum is identical for an electron with a given angular momentum in a given valley and an electron in the opposite valley and opposite angular momentum. This is a different way of saying that the system is time-reversal invariant. Let us therefore focus on the positive values of $m$ and $\tau$.

\section{Numerical Results and Discussion}

To obtain energy spectrum of a Dirac electron in the quantum dot we solve the eigenvalue equation (8) for a given angular momentum and valley index. The roots of this equation belong to the complex energy plane. The key observation is that the root finding problem in complex plane is not as straightforward as the root finding on the real axis. Therefore it is not straightforward to apply standard algorithms that are developed to search for roots on the real axis, and expect them to work in the complex plane too. Therefore here we adopt a simple and conservative, but slightly expensive method of scanning the whole complex plane of energy in order to make sure 


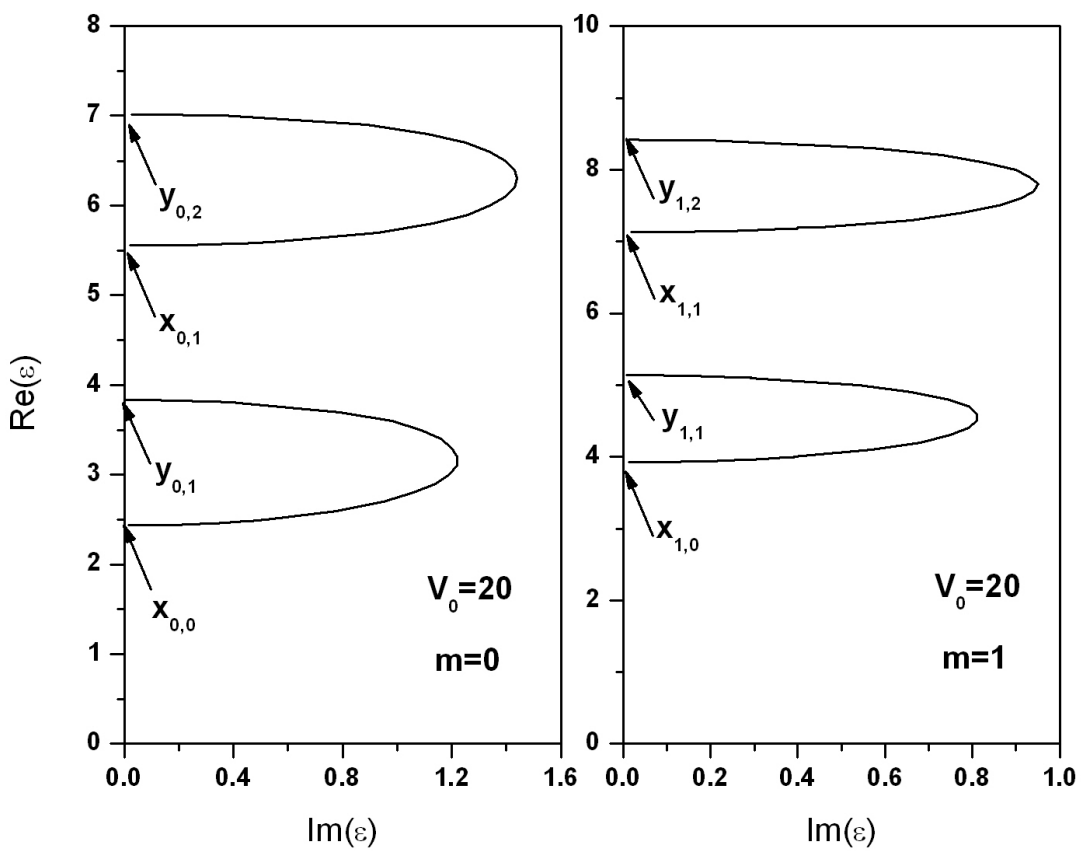

Figure 1. Two lowest energy bands of Dirac electrons in quantum dot for two angular momenta in the complex energy plane corresponding to $\nu_{0}=20$ and $\tau=1$.

no solutions are missed. Smaller imaginary times correspond to longer trapping time and the zero imaginary time correspond to infinitely long trapping time, i.e. non-decaying state.

In Fig. 1 we show results of our calculations far from the energies near the confinement potential edge and for $\tau=1$ and lowest values of $m$. As we can see from these data, instead of a single discrete point there is a continuum of solutions for the energy eigenvalue with different imaginary values. This is almost a "band" of energies which is quite counterintuitive for a quantum dot of finite size. The various "bands" corresponding to different choices of $(n, m)$ are however separated from one another band by energy gap. Within each band every energy state has different value of imaginary part which determines the broadening of state and trapping time of electron in the quantum dot. Even more interesting are the upper and lower edged of the bands that are denoted by $x_{m, n}$ and $y_{m, n}$ in this figure: These two values are $n$ 'th roots of the Bessel functions of order $m$ that are localized respectively inside and outside the QD. For electrons precisely tuned to these energies, one can realize the exact localization, as the imaginary part of energy vanishes at these energies. As can be seen in Fig. 1, by moving away from these "band edges" deep inside the band, the energy states at the middle of the energy bands have the typical imaginary part of the order 1 (in dimensionless units). Therefore, the trapping time for a quantum dot with $\mathrm{R}=50 \mathrm{~nm}$, is $\tau=\hbar R / a t \sim 10^{-13} s$. This is a relatively short time and we should consider the electrons in such energy states as weakly localized in quantum dot. Using the ordinary root finding methods one can only capture the state at the middle of the present band with maximal imaginary time, while a brute force scanning captures a band of energies.

To get a feeling for the way the roots $x_{m, n}$ of the $m$ th order Bessel function can possibly be a solution, let us examine if there is any solution around $\varepsilon=\nu_{0}$ where $\nu_{0}$ is the dimensionless 

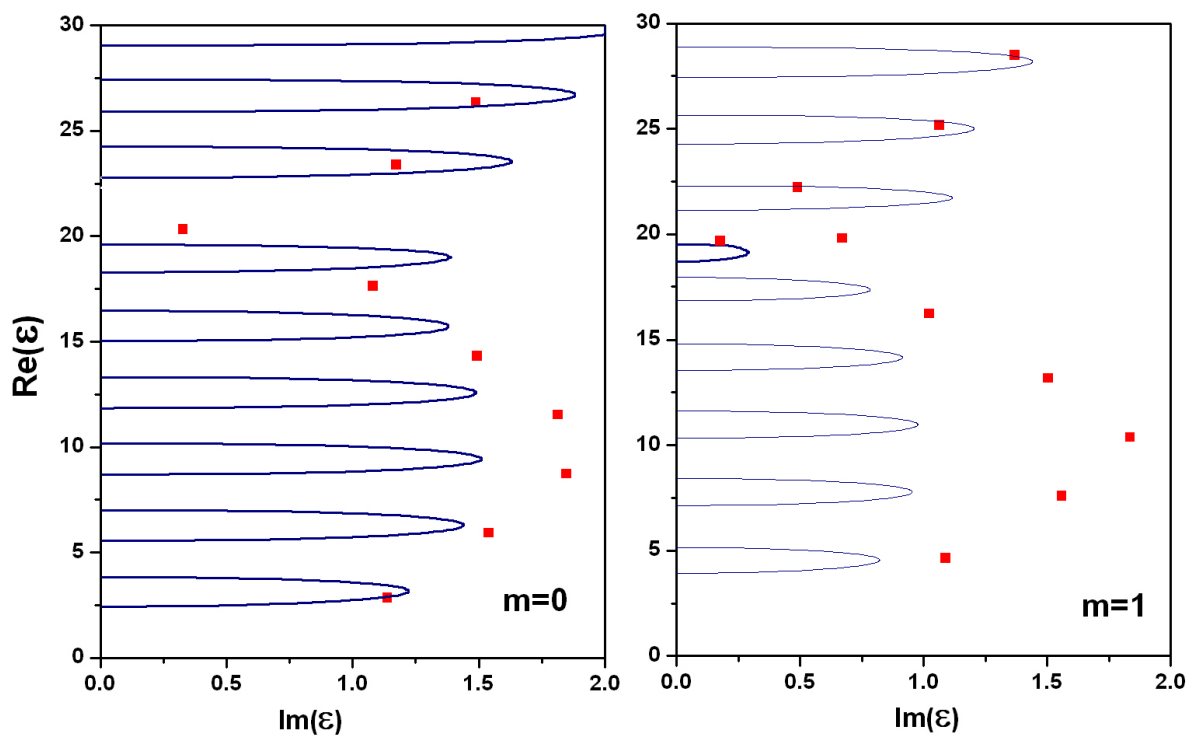

Figure 2. The energy spectrum of Dirac electrons in quantum dot for two angular momenta in complex energy plane for $\nu_{0}=20$ and $\tau=1$.

confining potential? In this case the Eq. (8) becomes,

$$
\frac{H_{|-\tau m|}^{(1)}(0)}{H_{|-\tau(m+1)|}^{(1)}(0)}=\frac{J_{|-\tau m|}\left(\nu_{0}\right)}{J_{|-\tau(m+1)|}\left(\nu_{0}\right)}
$$

The left hand side of this equation is zero, so that $J_{|-\tau m|}\left(\nu_{0}\right)=0 \Rightarrow \nu_{0}=x_{m, n}$. As pointed out the $x_{m, n}$ is $n$ 'th root of Bessel function $J_{m}$. However, the imaginary part of the energy at this particular state is zero, which implies that the electron at this energy is exactly localized in quantum dot. This result is consistent with results of Ref. [13] where it was shown that the electron can be strongly trapped in quantum dot when the energy of electron and confinement potential satisfy special conditions.

In Fig. 2 we give a comparison of our numerical results for the energy spectrum with that of Ref. [13]. The results of this reference are denoted with a square dot, while our results are denoted with continuous solid line. As can be seen the brute force method of scanning the complex plane for finding the solutions captures at some energy eigenvalues agrees with those obtained in Ref. [13], while it also captures a continuum of solutions. This figure shows that Dirac electrons energy spectrum consist of energy bands due to the relativistic properties and interference effect in the quantum dots. In addition to quasi-bound states with finite imaginary time there are also long lived bound states possible at the edges of these bands.

It is also possible to slightly move away from this (real) root and see the qualitative behavior of the complex eigenvalues of energy as the real part deviates from the particular solution at 
$\varepsilon=\nu_{0}$. For deviation $\delta \varepsilon$ from $\varepsilon=\nu_{0}$ solution the eigenvalue condition becomes,

$$
\frac{H_{m}^{(1)}(\delta \varepsilon)}{H_{m+1}^{(1)}(\delta \varepsilon)}=\frac{J_{m}\left(x_{m, n}+\delta \varepsilon+\delta \nu\right)}{J_{m+1}\left(x_{m, n}+\delta \varepsilon+\delta \nu\right)}
$$

where $\delta \varepsilon=\varepsilon-\nu_{0}, \delta \nu=\nu_{0}-x_{m, n}$ (for $m>0$ and $\tau=1$ ). Taylor expansion gives,

$$
\begin{cases}-\delta \varepsilon \ln \delta \varepsilon+i \frac{\pi}{2} \delta \varepsilon+(\delta \varepsilon+\delta \nu)=0 & m=0, \tau=1 \\ \frac{\delta \varepsilon}{2 m}+i \frac{\pi \delta \varepsilon^{2 m+1}}{2^{2 m+1}(m !)^{2}}+(\delta \varepsilon+\delta \nu)=0 & m>0, \tau=1\end{cases}
$$

where we used the relation $J_{m}^{\prime}\left(x_{m, n}\right)=-J_{m+1}\left(x_{m, n}\right)$. By solving this equation we obtain real and imaginary parts of energy as:

$$
\left\{\begin{array}{lrl}
\delta \varepsilon=\frac{\delta \nu}{\ln \delta \nu}+i \frac{\pi}{2} \frac{\delta \nu}{\ln \delta \nu} & m=0, \tau=1, \\
\delta \varepsilon=-\left(\frac{2 m}{2 m+1}\right) \delta \nu+i \frac{\pi}{2^{2 m+1}(m !)^{2}}\left(\frac{2 m}{2 m+1}\right)^{2(m+1)} \delta \nu & m>0, \tau=1 .
\end{array}\right.
$$

Now we can find the intrinsic broadening of energy bans for Dirac electrons in quantum dot as:

$$
\begin{cases}1 / \tau \propto \frac{\pi}{2} \frac{\delta \nu}{\ln \delta \nu} & m=0, \tau=1 \\ 1 / \tau \propto \frac{\pi}{2^{2 m+1}(m !)^{2}}\left(\frac{2 m}{2 m+1}\right)^{2(m+1)} \delta \nu & m>0, \tau=1\end{cases}
$$

This equation shows that broadening of energy levels depends on the orbital angular momentum of electron, index of quasi bound state and the depth of confinement potential at least for a one particular energy band developed from the $\varepsilon=\nu_{0}$ infinitely long lived state.

\section{Conclusion}

Spectrum of relativistic Dirac electrons in a quantum well instead of single isolated complex energy points, consists of bands of complex energies. Fine tunneling the energy one could realize a discrete set of non-leaking states that are not able to make use of Klein-tunneling to escape the QD. This opens of the possibility to reconsider Dirac quantum dots for applications in various electronic devices and nano-structures.

\section{References}

[1] Abergel D S L, Apalkov V, Berashevich J, Ziegler K and Chakraborty T 2010 Advances in Physics $59261-482$

[2] Downing C A, Stone D A and Portnol M E 2011 Phys. Rev. B 84155437

[3] Masir M R, Matulis A and Peeters F M 2009 Phys. Rev. B 79155451

[4] Roy P, Ghosh T and Bhattacharya K 2012 J. of Physics: Condens. Matter 24055301

[5] Matulis A 2012 Lithuanian Journal of Physics 52126141

[6] M Masir P Vasilopoulos F M Peeters 2011 J. of Physics: Condens. Matter 23315301

[7] Park P S, Kim S and Yang S R E 2010 Journal of Physics: Condens. Matter 22375302

[8] Masir M R, Matulis A and Peeters F M 2011 Phys. Rev. B 84245413

[9] Giavaras G and Nori F 2011 Phys. Rev. B 83165427

[10] Giavaras G and Nori F 2012 Phys. Rev. B 85165446

[11] Pal G, Apel W and Schweitzer L 2011 Phys. Rev. B 84075446

[12] Pieper A, Heinisch R L, Wellein G and Fehske H 2014 Phys. Rev. B 89165121

[13] Hewageegana P and Apalkov V 2008 Phys. Rev. B 77245426

[14] Hewageegana P and Apalkov V 2009 Phys. Rev. B 79115418

[15] Roslyak O, Gumbs G and Mukamel S 2012 J. Chem. Phys. 136194106 